\title{
Can phosphatidylinositol 3-kinase/mammalian target of rapamycin inhibition ERase them all?
}

\author{
Dominique S Meyer and Mohamed Bentires-Alj**
}

\begin{abstract}
Seventy percent of breast tumors are estrogen receptor (ER) positive. Although endocrine therapy is successful for the majority of patients with ER-positive tumors, approximately $30 \%$ show de novo or acquired resistance and the underlying molecular mechanisms and biomarkers that predict such resistance remain elusive. Two recent papers report that hyperactivation of the phosphatidylinositol 3-kinase (PI3K) pathway produces resistance to tamoxifen. This raises the possibility that combining endocrine therapy and PI3K inhibition may be more effective than monotherapy for treating ER-positive breast tumors, either as first-line therapy for tumors with high PI3K activity or after the development of resistance to endocrine therapy.
\end{abstract}

\section{Background}

Tamoxifen, which interferes with estrogen for receptor binding, was the first successful targeted therapy and has been the treatment of choice for estrogen receptor (ER)positive breast cancers for more than two decades. Unfortunately, resistance occurs in approximately $30 \%$ of patients and the mechanisms of resistance act directly on the ER pathway and/or activate parallel pathways that block the anti-proliferative and pro-apoptotic actions of tamoxifen [1,2]. For example, increased mitogenactivated protein kinase (MAPK) and phosphatidylinositol 3-kinase (PI3K) signaling downstream of growth factor receptors (for example, ERBB2 and Insulin-like growth factor 1 receptor (IGF-1R)) has been implicated in a crosstalk with ER and in resistance to endocrine therapy [1-3].

The PI3K pathway, a central regulator of diverse normal cellular functions, is very often subverted during neoplastic transformation and contributes to several

*Correspondence: bentires@fmi.ch

Friedrich Miescher Institute for Biomedical Research, Maulbeerstr. 66, 4058 Basel, Switzerland hallmarks of cancer that result in a competitive advantage for cancer cells [4-6]. Not surprisingly, the PI3K cascade is an attractive therapeutic target and several inhibitors are currently in phase I/II clinical trials. Preclinical studies have shown that PI3K inhibition circumvents resistance to trastuzumab in ERBB2-positive breast cancers and this has led to clinical trials combining antiERBB2 and anti-PI3K therapies [7]. Although PI3K has been implicated in resistance to endocrine therapy of ERpositive tumors, several questions remain unanswered. Is PI3K inhibition, alone or in combination with tamoxifen, effective in tamoxifen-resistant ER-positive cancer cells? Does PI3K inhibition block the emergence of tamoxifen resistance? Does a PI3K hyperactivation signature in human tumors predict tamoxifen sensitivity? Two recent papers now provide some answers to these outstanding questions.

\section{The articles}

Creighton and colleagues [8] investigated the relationship between the PI3K pathway and ER levels and activity using breast cancer cell lines and data sets of human breast tumors, and they defined proteomic and transcriptomic signatures of a hyperactivated PI3K pathway. These signatures negatively correlate with ER levels within ER-positive human breast tumors and are associated with the luminal B breast cancer subtype, the more aggressive subtype of ER-positive tumors. In addition, the transcriptomic signature was shown to predict the worst prognosis in ER-positive tumors. Treatment with the dual PI3K/mammalian target of rapamycin (mTOR) inhibitor BEZ235 increased ER expression in a number of ER-positive breast cancer cell lines. Interestingly, treatment of two luminal B ER-positive cell lines simultaneously with tamoxifen and BEZ-235 reduced cell proliferation more than with either inhibitor alone. Of note, luminal A tumors might also benefit from PI3K inhibition as the luminal A cell lines used by Creighton and colleagues [8] and by Miller and colleagues [9] are also sensitive to BEZ235.

Whether hyperactivation of the PI3K pathway contributes to tamoxifen resistance after chronic tamoxifen treatment was not addressed by this study, but was 
answered by Miller and colleagues [9], who generated estrogen-independent breast cancer cell lines from estrogen-dependent ER-positive lines by long-term estrogen deprivation (LTED). Unbiased phosphoprotein microarrays, immunocapture arrays and transcription profiling revealed an increase in PI3K/mTOR signaling as a consequence of higher phosphorylation of the insulin receptor and/or IGF-1R in LTED cells compared with parental cells. Inhibition of PI3K/mTOR using BEZ235 [10] reduced cell growth in both parental and LTED cells and increased apoptosis in most of them. It remains unclear whether LTED cells are more reliant on the PI3K pathway for survival than their parental counterparts as BEZ235 caused a similar level of apoptosis in the two lines. Finally, the authors found that the protein signature of activated PI3K signaling predicted disease outcome after adjuvant endocrine therapy [9].

\section{The viewpoint}

Both studies highlight the role of the PI3K pathway in resistance to tamoxifen. Creighton and colleagues [8] suggest that this effect is caused by PI3K-mediated downregulation of ER. This is also supported by the observation of Miller and colleagues [9] that PI3K pathway activation is inversely correlated with expression of ER in 64 human ER-positive breast tumors. As two lines (MCF7-LTED, HCC1428-LTED) used by Miller and colleagues showed a hyperactivated PI3K pathway compared with parental cells, but no decrease in ER expression, it is conceivable that further mechanisms account for PI3K-mediated resistance [9]. Altogether, the two studies suggest that targeting PI3K/mTOR and ER should be effective in ER-positive tumors and warrant complementary studies using in vivo models. Indeed, the interaction of cancer cells with the stroma might modulate estrogen sensitivity and lead to different mechanisms of resistance [2].

Although these studies might suggest that ER-positive tumors with a hyperactivated PI3K pathway should correlate with poor prognosis, this does not seem to be the case, at least for tumors showing activation of the PI3K pathway driven by PIK3CA mutations. Indeed, three recent studies reported that mutations of PIK3CA, in particular the catalytic domain mutation H1047R, are associated with better prognosis in ER-positive breast cancers [11-13]. This is surprising given the transforming effect of PIK3CA mutations in experimental systems. One possible explanation for this 'PIK3CA paradox' is that most experimental models assess effects on primary tumor growth and not metastases and long-term survival. Alternatively, PIK3CA mutations might only moderately activate the pathway and/or constitutive activity of mutant PIK3CA may induce negative feedback loops that preclude a more pronounced activation of the pathway [14]. In fact, several cell lines with PIK3CA mutations (MCF-7, BT-483, MB-361) [15] were associated with a low/intermediate PI3K score in the proteomic signature of Creighton and colleagues [8]. Therefore, better experimental models and systems biology-like assessment of the PI3K network, including the activities of all intermediates of the pathway, associated feedback loops and collaborating oncogenic pathways, may be needed to solve the 'PIK3CA paradox'.

In summary, these two studies suggest hyperactivation of PI3K as a mechanism of resistance to tamoxifen, which may have clinical impact. It is reasonable to conclude that the combination of endocrine therapy and inhibition of PI3K in ER-positive tumors with a hyperactive PI3K pathway may be more effective than monotherapy. Studies on in vivo breast cancer models with alterations in the PI3K pathway should increase our understanding of this complex but fascinating signaling network, test the validity of this speculation and optimize combination therapies. Ultimately, clinical trials testing combinations of anti-ER and anti-PI3K therapies may finally lead to relapse-free therapy for patients with ER-positive tumors.

\section{Abbreviations}

ER, estrogen receptor; IGF-1R, Insulin-like growth factor 1 receptor; LETD, long-term estrogen deprivation; MTOR, mammalian target of rapamycin; PI3K, phosphatidylinositol 3-kinase.

Competing interests

The authors declare that they have no competing interests.

\section{Acknowledgements}

We thank various colleagues for helpful comments on the manuscript. Research in the lab of MB-A is supported by the Novartis Research Foundation, the European Research Council (ERC starting grant 243211-PTPsBDC), the Swiss Cancer League and the Krebsliga Beider Basel.

\section{Published: 20 October 2010}

\section{References}

1. Shou J, Massarweh S, Osborne CK, Wakeling AE, Ali S, Weiss H, Schiff R: Mechanisms of tamoxifen resistance: increased estrogen receptor-HER2/ neu cross-talk in ER/HER2-positive breast cancer. J Nat/ Cancer Inst 2004, 96:926-935.

2. Musgrove EA, Sutherland RL: Biological determinants of endocrine resistance in breast cancer. Nat Rev Cancer 2009, 9:631-643.

3. Miller TW, Pérez-Torres M, Narasanna A, Guix M, Stål O, Pérez-Tenorio G, Gonzalez-Angulo AM, Hennessy BT, Mills GB, Kennedy JP, Lindsley CW, Arteaga CL: Loss of Phosphatase and Tensin homologue deleted on chromosome 10 engages ErbB3 and insulin-like growth factor-I receptor signaling to promote antiestrogen resistance in breast cancer. Cancer Res 2009, 69:4192-4201.

4. Cantley LC: The phosphoinositide 3-kinase pathway. Science 2002, 296:1655-1657.

5. Bader AG, Kang S, Zhao L, Vogt PK: Oncogenic PI3K deregulates transcription and translation. Nat Rev Cancer 2005, 5:921-929.

6. Engelman JA, Luo J, Cantley LC: The evolution of phosphatidylinositol 3-kinases as regulators of growth and metabolism. Nat Rev Genet 2006, 7:606-619.

7. Junttila TT, Akita RW, Parsons K, Fields C, Lewis Phillips GD, Friedman LS, Sampath D, Sliwkowski MX: Ligand-independent HER2/HER3/PI3K complex is disrupted by trastuzumab and is effectively inhibited by the PI3K inhibitor GDC-0941. Cancer Cell 2009, 15:429-440.

8. Creighton CJ, Fu X, Hennessy BT, Casa AJ, Zhang Y, Gonzalez-Angulo AM, 
Lluch A, Gray JW, Brown PH, Hilsenbeck SG, Osborne CK, Mills GB, Lee AV, Schiff R: Proteomic and transcriptomic profiling reveals a link between the PI3K pathway and lower estrogen-receptor (ER) levels and activity in ER+ breast cancer. Breast Cancer Res, 12:R40.

9. Miller TW, Hennessy BT, Gonzalez-Angulo AM, Fox EM, Mills GB, Chen H, Higham C, Garcia-Echeverria C, Shyr Y, Arteaga CL: Hyperactivation of phosphatidylinositol-3 kinase promotes escape from hormone dependence in estrogen receptor-positive human breast cancer. J Clin Invest, 120:2406-2413.

10. Maira SM, Stauffer F, Brueggen J, Furet P, Schnell C, Fritsch C, Brachmann S, Chène P, De Pover A, Schoemaker K, Fabbro D, Gabriel D, Simonen M, Murphy L, Finan P, Sellers W, García-Echeverría C: Identification and characterization of NVP-BEZ235, a new orally available dual phosphatidylinositol 3-kinase/ mammalian target of rapamycin inhibitor with potent in vivo antitumor activity. Mol Cancer Ther 2008, 7:1851-1863.

11. Loi S, Haibe-Kains B, Majjaj S, Lallemand F, Durbecq V, Larsimont D, GonzalezAngulo AM, Pusztai L, Symmans WF, Bardelli A, Ellis P, Tutt AN, Gillett CE, Hennessy BT, Mills GB, Phillips WA, Piccart MJ, Speed TP, McArthur GA, Sotiriou C: PIK3CA mutations associated with gene signature of low mTORC1 signaling and better outcomes in estrogen receptor-positive breast cancer. Proc Natl Acad Sci U S A, 107:10208-10213.
12. Kalinsky K, Jacks LM, Heguy A, Patil S, Drobnjak M, Bhanot UK, Hedvat CV, Traina TA, Solit D, Gerald W, Moynahan ME: PIK3CA mutation associates with improved outcome in breast cancer. Clin Cancer Res 2009, 15:5049-5059.

13. Ellis MJ, Lin L, Crowder R, Tao Y, Hoog J, Snider J, Davies S, DeSchryver K, Evans DB, Steinseifer J, Bandaru R, Liu W, Gardner H, Semiglazov V, Watson M, Hunt $\mathrm{K}$, Olson J, Baselga J: Phosphatidyl-inositol-3-kinase alpha catalytic subunit mutation and response to neoadjuvant endocrine therapy for estrogen receptor positive breast cancer. Breast Cancer Res Treat, 119:379-390.

14. Li J, DeFea K, Roth RA: Modulation of insulin receptor substrate-1 tyrosine phosphorylation by an Akt/phosphatidylinositol 3-kinase pathway. J Biol Chem 1999, 274:9351-9356.

15. Hollestelle A, Elstrodt F, Nagel JH, Kallemeijn WW, Schutte M: Phosphatidylinositol-3-OH kinase or RAS pathway mutations in human breast cancer cell lines. Mol Cancer Res 2007, 5:195-201.

doi:10.1186/bcr2718

Cite this article as: Meyer DS, Bentires-Alj M: Can phosphatidylinositol

3-kinase/mammalian target of rapamycin inhibition ERase them all? Breast Cancer Research 2010, 12:315. 